\title{
A case of spontaneous cerebellar hemorrhage
}

\author{
Arash Lashani* and Robert Hage \\ Department of Anatomical Sciences, St. George's University, West Indies
}

\begin{abstract}
Intracerebral hemorrhage $(\mathrm{ICH})$ is a rare phenomenon for which $10 \%$ occur in the cerebellum. They occur in older adults and are associated with hypertension in the majority of cases. They have been reported in adolescents and young adults in association with arteriovenous malformations and angiomas. Spontaneous cerebellar hemorrhaging, in the absence of trauma or any identifiable well-established risk factors, is an exceedingly rare occurrence, with a mortality ranging from $25-57 \%$. A case of a 26-year-old male with a spontaneous cerebellar hemorrhage is presented. He is a healthy young adult without identifiable risk factors for cerebrovascular accident (CVA) apart from marijuana use, which has not definitively been established as a risk factor. Several possible etiology's are explored, and after nine years his post-event status is assessed.
\end{abstract}

\section{Introduction}

Spontaneous cerebellar hemorrhage is defined as occurring without prior warning, trauma or external cause, and an absence of identifiable risk factors. Cerebellar hemorrhage, among other types of intracerebral hemorrhage (ICH), may be classified as a hemorrhagic stroke. Stroke is defined as an interruption to the blood supply of the brain, which results in insufficient oxygen delivery to brain tissue. According to the American Heart Association [1], the prevalence of stroke in the United States is around $2.5 \%$ and affects approximately 7 million Americans, with more than $90 \%$ of these cases attributable to modifiable risk factors. ICH may be due to trauma, underlying pathology, or it may be spontaneous. It can be classified further according to the region of the brain involved. Hemorrhaging originating in the cerebellum makes up nearly $10 \%$ of the cases of all ICH [2]. The first reported operation on a cerebellar hemorrhage was in 1906 according to Ott, et al. [3]. Since then, more reports and research have brought further attention and knowledge to this pathology. Studies have reported that cerebellar infarction accounts for almost $3 \%$ of all strokes, about 20,000 new cerebellar infarctions each year in the United States [4]. Worldwide incidence has increased in young adults over the past three decades, with strokes of undetermined etiology accounting for up to $30 \%$ of cases [5]. Non-traumatic cerebellar hemorrhage affects approximately 10,000 patients in the United States each year [6], with the majority encompassing older adults due to an identifiable chronic condition. However, spontaneous cerebellar hemorrhaging in healthy young adults with no underlying medical conditions and risk factors, is rare.

The posteroinferior aspect of the skull contains the brainstem and cerebellum. The cerebellum is composed of two lateral hemispheres and a midline vermis which are considered important for coordinating movements and organizing visuospatial information. Damage to the cerebellum produces deficits that are ipsilateral to the region affected [4]. Three paired arteries supply the cerebellum; the posterior inferior cerebellar artery (PICA), the anterior inferior cerebellar artery (AICA), and the superior cerebellar artery (SCA). These three arteries are part of the posterior circulation of the brain, known as the vertebrobasilar system [4]. This vasculature supplies the cerebellum, and its very specific micro-circuitry so that it can perform many complex functions. As well, the cells of the cerebellar cortex possess a uniform laminar arrangement, creating a specific orientation and running of the nerve fibers to and from the cerebellum. This is in contrast with the orientation of nerve fibers running through the cortex and other parts of the brain. Although it is generally well established that the cerebellum is responsible for posture, voluntary movement, goal-directed and spontaneous movements [7], its specific role in processing relevant spatial information remains elusive. Molinari and Leggio mention that even though cerebellar damage has been reported to impair spatial cognition, "response patterns have been very different from damage to classical spatial brain structures such as the hippocampus and parietal cortex" [8]. Damage to the cerebellum does not produce predictable deficits as does damage to other brain structures. Patients with a cerebellar infarction may present with a wide array of deficits and impairments, ranging from minor maladies to life-threatening situations. Although the case that will be presented occurred in Canada, it is relevant as many citizens of the Caribbean islands have connections and ties to the US and Canada and travel back and forth.

\section{Case report}

A 26-year-old previously healthy male was assisted into a hospital in the greater Toronto area on a winter's day in 2011. During triaging in the emergency room he was vomiting incessantly, appeared to be lethargic with eyes fixed shut and had slurred speech. An intravenous (IV) line with dimenhydrinate in normal saline ceased his emesis. Routine workup was unremarkable. A head CT with contrast showed cerebellar attenuation in the area of the right cerebellar lobe, consistent with a cerebellar hematoma, which led to an interfacility transfer to a hospital with neurosurgical facilities (Figure 1).

During the course of the patient's stay in the ICU, a thorough history was obtained. According to the patient, it all began as follows; while lying in bed with a face down position, he turned his head to the

${ }^{\star}$ Correspondence to: Arash Lashani, Department of Anatomical Sciences, St George’s University, West Indies, Tel: 16476923326; E-mail: alashani@sgu.edu

Key words: intracerebral hemorrhage, cerebellar hemorrhage, trauma, hematoma

Received: April 27, 2020; Accepted: May 12, 2020; Published: May 15, 2020 


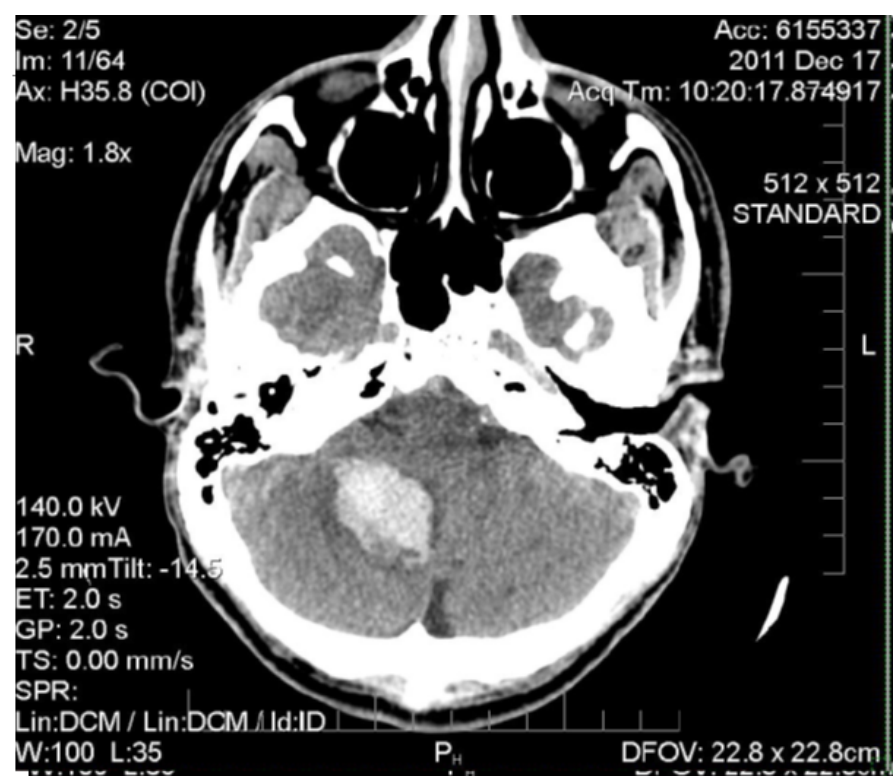

Figure 1. Unenhanced axial CT scan of the head. There is a $25 \times 28 \times 33 \mathrm{~mm}$ hyperdense, acute parenchymal haemorrhage in the right cerebellar hemisphere causing herniation of the right cerebellar tonsil into the foramen magnum with partial effacement of the fourth ventricle and early hydrocephalus. The etiology of the haemorrhage is nonspecific based on imaging findings and could be related to haemorrhagic transformation of ischemic infarct, or haemorrhage into a neoplasm or cavernous malformation

side to view something. Upon doing so he felt a strange sensation pass through the back of his neck. He did not think much of it at the time, as it felt like a neck "cramp". However, over the course of 15 minutes; it rapidly changed for the worse. After the strange neck sensation, he began to feel a vague sense of uneasiness in his stomach and bowels; he thought it might be alleviated during a bowel movement. He was unsuccessful in passing stool, however the vague feeling of uneasiness turned into full-blown nausea, and he began to feel as if the world was spinning. He became concerned and called Ontario's telehealth hotline. Telehealth Ontario is a phone hotline available to residents that provides medical advice from registered nurses. After reporting his symptoms, a visit to the nearest hospital emergency room was advised. At this point he began vomiting uncontrollably, and the pronounced dizziness made it extremely difficult to keep his eyes open. Within half an hour of his initial symptoms he was driven to the hospital where he collapsed on the pavement.

A detailed report of his past medical history a few days after admission mentioned no identifiable risk factors, such as: history of hypertension, coagulopathy, head trauma, known family history of intracranial aneurysm or arteriovenous malformations (AVM), history of malignancy or use of drugs (e.g. cocaine and amphetamines) [2]. He regularly used marijuana and had smoked the night before, but not the morning of. As marijuana was still illegal in Canada at the time, the only means of procurement was through illicit avenues, which raises suspicion as to the source and processing of the cannabis. Of particular interest is the following statement: "That night I felt uneasy when I was procuring the marijuana, because it was from a different supplier. I noticed it burned differently then it usually did that night, being much moister then I ever recalled". No drug screening was performed.

The patient's hospital stay was uneventful and managed medically. During his stay in the ICU, the patient received an MRI for more detailed imaging, which demonstrated the extent of the hematoma (Figure 2 and 3 ).
The MRI did not reveal further pathology. He stayed for one week in the ICU, followed by several days on the general ward, and ultimately became wheelchair bound on discharge from the hospital. The patient remained wheelchair bound due to an unsteady gait for three weeks. Physiotherapy was recommended, but not utilized as it required out-ofpocket costs. A cerebral angiogram was recommended, but the patient refused due to concerns about the perceived risks. The neurosurgical team therefore recommended an angiogram in $6-12$ months after discharge, which could potentially reveal a vascular lesion, imposing risks in the future. An angiogram one year after discharge revealed evidence of a non-aggressive slow flowing dural arteriovenous fistula, described as an incidental finding and most likely not related to the patient's past presentation. A second cerebral angiogram one year from the date of the first was completed to track any potential changes

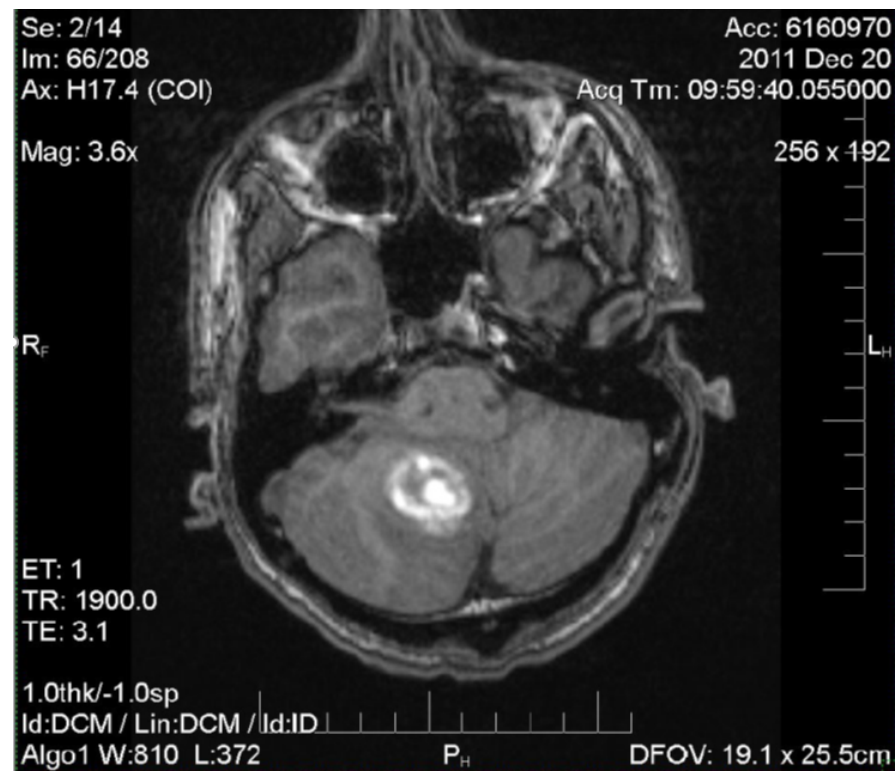

Figure 2. MRI: Nonspecific right inferomedial cerebellar hemisphere intra-parenchyma hematoma measuring $2.8 \mathrm{~cm}$. Mild local mass effect on the inferior fourth ventricle. No obstructive hydrocephalus. No underlying lesion identified at this time

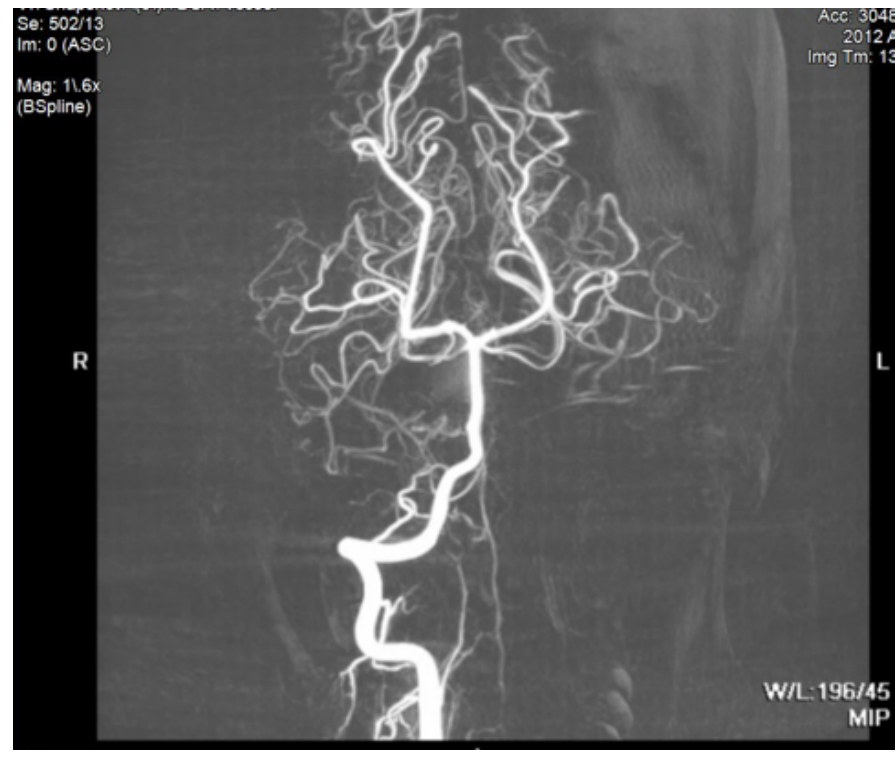

Figure 3. MR angiogram is unremarkable 
in vasculature, ultimately showing no changes, and the patient was unconditionally released from the team's care.

Recovery from an ICH varies greatly among individuals and can be very difficult to predict early on during the acute phases. Follow up with the patient in 2020, 9 years post-event, revealed a 35 -year-old male who had recovered well. His speech was fluid and coherent; however, he lost his balance easily and could not perform heel-to-toe walk without falling over. His vitals were all within normal range. The only medication he was on were loratadine or diphenhydramine for allergies as needed. According to the patient, he experiences tremors at rest which occasionally become intentional when stressed. There are bouts of extreme vertigo that cause him to roll around and fall towards the ground, as he feels as though he loses all motor control. A Dix-Hallpike test is negative. His balance is easily compromised, especially on the right side, however, he has otherwise made a remarkable recovery given the circumstances. He still uses marijuana occasionally.

\section{Discussion}

The majority of cerebellar hemorrhages occur in older adults with hypertension (60-70\% of cases), while the remainder are largely due to arteriovenous malformations (AVM), bleeding diathesis, and tumors [9]. Other causes of cerebellar hemorrhage include trauma, coagulopathy, ischemic stroke with hemorrhagic transformation, cerebral amyloid angiopathy, septic embolism, encephalitis, vasculitis, sympathomimetic drugs (e.g. cocaine, amphetamines) and aneurysm of the posterior cerebral circulation. If a tumor is the cause of bleeding, it consistently is a glioblastoma [2]. A cerebellar hemorrhage secondary to an AVM tends to occur in younger individuals, whereas those caused by vascular disease secondary to hypertension is more commonly seen in older individuals [10]. Symptom presentation varies widely according to the location and size of the hemorrhage [3]. Smaller hemorrhages may not alter the patient's mental status, whereas larger ones may leave the patient in a stupor or unresponsive. Commonly reported symptoms for cerebellar hemorrhages include abrupt onset headache, nausea and vomiting, truncal ataxia, vertigo, dysarthria, nuchal rigidity and an altered mental status or level of consciousness [2]. This is of note because the patient under study did not complain of headaches.

Cerebellar hemorrhage falls under ICH management guidelines [11]; however, there is a great deal of clinical experience that plays a factor in determining how the guidelines are applied. According to Little, et al. this especially applies during cases of brainstem compression or large hematomas with mass effect, resulting in acute hydrocephalus [12]. In these cases, non-operable patients had a grave prognosis. Nonetheless, there is a lack of evidence supporting surgical management of patients with ICH leading to an overall decreased mortality, according to CLEAR III and MISTIE III trials. Removal of large hematomas is not necessarily associated with improved functional outcomes [6].

In this case the neurosurgical team decided against surgical treatment, a decision based on their clinical experience. The decision to undergo nonsurgical management in our patient who presented with a cerebellar hematoma with partial effacement of the fourth ventricle and partial herniation through the foramen magnum is debatable. The mortality associated with cerebellar hemorrhage can range from $25-57 \%$ and stems largely from complications such as brainstem compression, cerebellar herniation and compression of the fourth ventricle with ensuing obstructive hydrocephalus [2].

The vast majority of cerebellar hemorrhages have identifiable causes or predisposing risk factors. Only a fraction of the cases of cerebellar hemorrhage are truly spontaneous in that there is no history of trauma, identifiable cause, or predisposing risk factors. Dayes, et al. report the cases of 3 young male adults aged 30, 38 and 27 years of age which revealed a medulloblastoma, hemophilia $\mathrm{A}$ and malignant melanoma respectively [9]. Two of the three cases involved a tumor, and one involved a bleeding disorder, all identified as risk factors. Mitchell \& Angrist studied 12 cases of death caused by cerebellar hemorrhage [13]. All of them had identifiable risk factors including malignant nephrosclerosis, cerebral arteriosclerosis and myelogenous leukemia, with the most common risk factor being hypertension related cardiovascular disease. Hyland and Levy identified 32 cases of cerebellar hemorrhage, four of which an underlying lesion was not demonstrated. The authors hypothesize that the lesions were so small they essentially bled out and were compressed [14].

A review of the literature revealed one other potential association that requires consideration, namely, marijuana. The major psychoactive ingredient in marijuana is tetrahydrocannabinol (THC), a lipid soluble substance with a high volume of distribution. Consuming one marijuana joint or cigarette can deliver between 2.5 to $5 \mathrm{mg}$ of THC. There are many other substances found in marijuana, including cannabidiol (CBD) and they all have varying effects on the central nervous system which include sedation, analgesia and anticonvulsant properties. The literature on cannabis use is equivocal, with reports of neuroprotective effects from oxidant injury to other benefits such as analgesia, ocular hypotension and antiemetic properties [15]. Marijuana use is associated with systemic hypotension, impaired peripheral vasomotor reflexes, altered cerebral blood flow and cerebral vascular autoregulation [15]. An association between marijuana use and stroke in adults has also been reported, although the mechanism and nature of that relationship remains unclear. One hypothesis is that marijuana causes vascular injury by promoting vasospasm, especially in infrequent users. According to a Nationwide Inpatient Sample database from 2004-2011 [5], the incidence of acute ischemic stroke in marijuana users was $0.4 \%$ among those aged 15-54 years. With marijuana legalization in places like Canada, its use is even more prevalent. Therefore, one would expect to see more cases, but only a small number of cerebellar infarctions in users is found globally. One possible explanation is there might be confounding variables, such as contamination of marijuana with other substances [15].

The literature does not provide a definitive answer in regards to the association between acute ischemia and marijuana use. Two prospective trials did not show an association between marijuana use and stroke. One case-controlled study found a 2-3 times increased odds of stroke in marijuana users, determined to be due to the confounding variable of tobacco use associated with marijuana smoking. Although. a national database study did find a 1-17 times increased odds of stroke associated with marijuana use even after controlling for tobacco use [5]. Thus, there is no definitive support of the hypothesis that marijuana use is associated with strokes in young adults. Our patient used marijuana daily for many years prior, but not on the morning of the incident.

Cannabis and its associated products can become contaminated with pesticides, molds, bacteria, metals, and solvents [16,17]. Due to marijuana being illegal in many countries and states in America, there is the likelihood that marijuana can be cross-contaminated with cocaine or amphetamines, as many suppliers deal with more than one illicit substance. Cannabis plants and products can pick up molds or bacteria while growing, and may accumulate high levels of metal from the soil or growth medium. Contamination by fertilizers can also occur. Production of cannabis related products such as concentrates 
(e.g. shatter, batter, budder), present further opportunity for processing agents, such as butane and propane, to present health problems [16]. Illicitly obtained marijuana is not controlled and there are no regulatory standards as to filtration. It is possible that in this case, the marijuana the patient used the night before was contaminated.

\section{Conclusion}

Spontaneous cerebellar hemorrhage in young healthy adults is an exceedingly rare phenomenon that requires further investigation and research. Although a causal pathway may never be elucidated, rapid recognition and treatment of a cerebellar hemorrhage can make the difference between life and death. Guidelines on management of ICH exist, but are not well defined for cerebellar hemorrhage. Since each case is unique, leniency in strictly following the guidelines should be expected. Further research may reveal causes and underlying pathophysiology. More research between marijuana use, additives and stroke is required to prove or refute the association with $\mathrm{ICH}$, in particular cerebellar hemorrhage, which has a high mortality rate.

\section{References}

1. Benjamin EJ, Muntner P, Alonso A, Bittencourt MS, Callaway CW, et al. (2019) Heart disease and stroke statistics-2019 update: A report from the American heart association. Circulation 139: e56-e528.

2. Fischer MA, Das JM (2019) Cerebellar hematoma. StatPearls [Internet].

3. Ott KH, Kase CS, Ojemann RG, Mohr JP (1974) Cerebellar haemorrhage: diagnosis and treatment. Archives of Neurology 31: 160-167.

4. Edlow JA, Newman-Toker DE, Savitz SI (2008) Diagnosis and initial management of cerebellar infarction. The Lancet Neurology 7: 951-964.
5. Viswanathan V, Yu C, Sambursky JA, Kaur S, Simpkins AN (2019) Acute cerebral ischemia temporally associated with marijuana use. Cureus 11: e5239.

6. Hemphill JC, Amin-Hanjani S (2019) Cerebellar Intracerebral Haemorrhage. JAMA 322: $1355-1356$.

7. Middleton FA, Strick PL (1998) The cerebellum: an overview. Trends in Cognitive Sciences 2: 305-306

8. Molinari M, Leggio MG (2007) Cerebellar information processing and visuospatial functions. The Cerebellum 6: 214-220.

9. Dayes LA, Purtzer TJ, Shahhal I, Cojocaru T, Knierim D, et al. (1986) Acute spontaneous cerebellar haemorrhage. Journal of the National Medical Association 78: 495-499.

10. Odom GL, Tindall GT, Dukes HT (1961) Cerebellar hematoma caused by angiomatous malformations. Journal of Neurosurgery 18: 777-782.

11. Hemphill JC, Greenberg SM, Anderson CS, Becker K, Bendok BR, et al. (2015) Guidelines for the management of spontaneous intracerebral haemorrhage. Stroke 46: 2032-2060.

12. Little JR, Tubman DE, Ethier R (1978) Cerebellar haemorrhage in adults: diagnosis by computerized tomography. Journal of Neurosurgery 48: 575-579.

13. Mitchell N, Angrist A (1942) Spontaneous cerebellar haemorrhage: Report of fifteen cases. The American Journal of Pathology 18: 935-953.

14. Hyland HH, Levy M (1954) Spontaneous cerebellar haemorrhage. Canadian Medical Association Journal 71: 315-323.

15. Geller T, Loftis L, Brink DS (2004) Cerebellar infarction in adolescent males associated with acute marijuana use. Pediatrics 113: 365-370.

16. Seltenrich N (2019) Cannabis contaminants: Regulating solvents, microbes, and metals in legal weed. Environmental Health Perspectives 127: 0820011-0820016.

17. https://data.worldbank.org/indicator/BX.TRF.PWKR.DT.GD.ZS?locations=GD

Copyright: $\odot 2020$ Lashani A. This is an open-access article distributed under the terms of the Creative Commons Attribution License, which permits unrestricted use, distribution, and reproduction in any medium, provided the original author and source are credited. 